\title{
Cardiomiopatía hipertrófica en fase de burnout
}

\section{Hypertrophic cardiomyopathy in burnout phase}

Alejandro Echavarría-Cross ${ }^{1 凶} \underline{\text { ORCID }} \underline{\text { CVLAC }}$, Luis Guillermo Pájaro-Franco $2 \llbracket \underline{\text { ORCID }} \underline{\text { CVLAC }}$, Sebastián

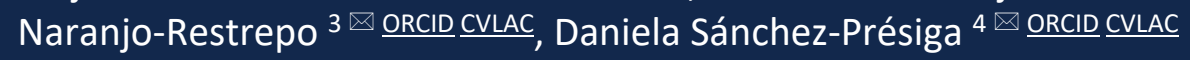

${ }^{1}$ Médico, residente de Medicina Interna, Universidad CES; Medellín, Colombia.

2 Médico, residente de Medicina Interna, Universidad de Cartagena; Cartagena, Colombia.

${ }^{3}$ Médico internista-cardiólogo, Hospital General de Medellín, CardioCES; Medellín, Colombia.

${ }^{4}$ Médica general, IPS Universitaria Clínica León XIII; Medellín, Colombia.

Fecha correspondencia:

Recibido: mayo 12 de 2021.

Revisado: septiembre 13 de 2021.

Aceptado: septiembre 24 de 2021

Forma de citar:

Echavarría-Cross A, Pájaro-Franco LG, Naranjo-Restrepo S, SánchezPrésiga D. Cardiomiopatía hipertrófica en fase de burnout. Rev CES Med. 2021; 35(3): 305-

315. https://dx.doi.org/10.21615/ cesmedicina. 6244

\section{Open access}

(C) Derecho de autor

Licencia creative commons

Ética de publicaciones

Revisión por pares

Gestión por Open Journal System DOI: 10.21615/cesmedicina.6244 ISSNe 2215-9177

ISSN 0120-8705

Publica con nosotros

\section{Resumen}

La cardiomiopatía hipertrófica es una entidad de origen genético que se caracteriza por hipertrofia ventricular izquierda en ausencia de otras enfermedades como etiología. Los pacientes suelen ser asintomáticos; sin embargo, el espectro de presentación es amplio pudiéndose encontrar falla cardiaca, obstrucción del tracto de salida, arritmias, muerte súbita y la etapa final o "burnout". La valoración morfológica inicial se realiza con ecocardiograma transtorácico, aunque la resonancia magnética cardiaca es el estudio de elección para confirmar el diagnóstico. Presentamos el caso de una mujer de 43 años con antecedente de cardiomiopatía hipertrófica quien había sido intervenida previamente e ingresó al servicio de urgencias de forma descompensada, documentándose progresión a fase "burnout" y su manejo subsecuente. Si bien este fenotipo no es común tiene importantes implicaciones pronósticas y en la calidad de vida. Por lo anterior, un diagnóstico y tratamiento oportuno pueden impactar favorablemente.

Palabras clave: cardiomiopatía hipertrófica; fase burnout; estudio de caso; imagen por resonancia magnética; fibrilación auricular. 


\section{Abstract}

Hypertrophic cardiomyopathy is a genetic entity characterized by left ventricular hypertrophy in the absence of other diseases as etiology. Patients are usually asymptomatic; however, the spectrum of presentation is wide and may include heart failure, outflow tract obstruction, arrhythmias, sudden death and the final stage also known as "burnout phase". The initial morphological assessment is performed with transthoracic echocardiography; however, cardiac magnetic resonance imaging is the gold standard to confirm the diagnosis. We present the case of a 43-year-old woman with a history of previously operated hypertrophic cardiomyopathy who was admitted to the emergency department decompensated, documenting progression to the "burnout" phase and her management. Although this phenotype is not common, it has prognostic and quality of life implications. Therefore, timely diagnosis and treatment can have an impact on these variables.

Keywords: cardiomyopathy, hypertrophic; burnout stage; case study; magnetic resonance imaging; atrial fibrillation.

\section{Introducción}

La cardiomiopatía hipertrófica es una condición en la cual el ventrículo izquierdo se hipertrofia, por definición en ausencia de otras causas (enfermedad primaria) ${ }^{(1,2)}$. A nivel mundial existen alrededor de 20 millones de personas con este diagnóstico, con una prevalencia que tiende al alza, cercana a 1500 en población general ${ }^{(1,3)}$, dado por el auge de la resonancia magnética cardiaca.

El fenotipo de burnout, también conocido como etapa terminal, es una presentación poco común en donde se presenta adelgazamiento de la pared con dilatación de las cuatro cavidades ${ }^{(1)}$. La reducción de la clase funcional NYHA (siglas del inglés New York Heart Association) y los síntomas congestivos son comunes en esta etapa y por lo tanto el tratamiento va encaminado a manejar la falla cardiaca, las arritmias y mejorar la calidad de vida ${ }^{(4,5)}$.

La relevancia de documentar este padecimiento radica en realizar un diagnóstico oportuno que permita iniciar un tratamiento encaminado a mejorar la calidad de vida del paciente. Presentamos el caso de mujer con antecedente de cardiomiopatía hipertrófica manejada 11 años atrás con miectomía septal, quien presentó descompensación de falla cardiaca y se documentó progresión al fenotipo de burnout. Posteriormente, se discuten los métodos diagnósticos y terapéuticos para estos pacientes. 


\section{Descripción del caso}

Mujer de 43 años, con antecedente de cardiomiopatía hipertrófica y comunicación interauricular, diagnosticada a los 32 años. En ese entonces debutó por un cuadro de falla cardiaca aguda, clase funcional IV de la New York Heart Association (NYHA), donde se evidenció hipertrofia septal, obstrucción del tracto de salida del ventrículo izquierdo, movimiento anterior sistólico de la válvula mitral y comunicación interauricular. Se le realizó miectomía septal, cierre del cortocircuito y reemplazo valvular mitral con prótesis mecánica; desde entonces, está anticoagulada con warfarina. La función sistólica biventricular postquirúrgica era normal y no presentaba dilatación de cavidades.

En esta ocasión, 11 años luego del diagnóstico, consultó a hospital de primer nivel por cuadro clínico de un mes de evolución, consistente en edema de miembros inferiores, palpitaciones y disnea severa presente incluso durante el reposo. Ante la sospecha clínica de falla cardiaca aguda, solicitan un electrocardiograma que evidenció fibrilación auricular con respuesta ventricular rápida e inician manejo antiarrítmico, control de frecuencia y diurético. Al no contar con paraclínicos especializados ni imágenes diagnósticas avanzadas, administraron metoprolol vía oral, amiodarona y furosemida intravenosas, y fue trasladada a una institución de tercer nivel de complejidad.

Al ingreso se encontraba en mal estado general, somnolienta, con franca dificultad respiratoria, presión arterial de 100/70 mm Hg, frecuencia cardiaca de 100 latidos por minuto, saturación de oxígeno $76 \%$. Al examen físico se encontró anasarca, ingurgitación yugular, ruidos cardiacos arrítmicos y crépitos bilaterales pulmonares. Ante la posibilidad de disfunción de la válvula protésica se solicitó un ecocardiograma transtorácico y un nuevo electrocardiograma, evidenciándose ritmo de fibrilación auricular con respuesta ventricular rápida y bloqueo de rama izquierda (figura 1). 


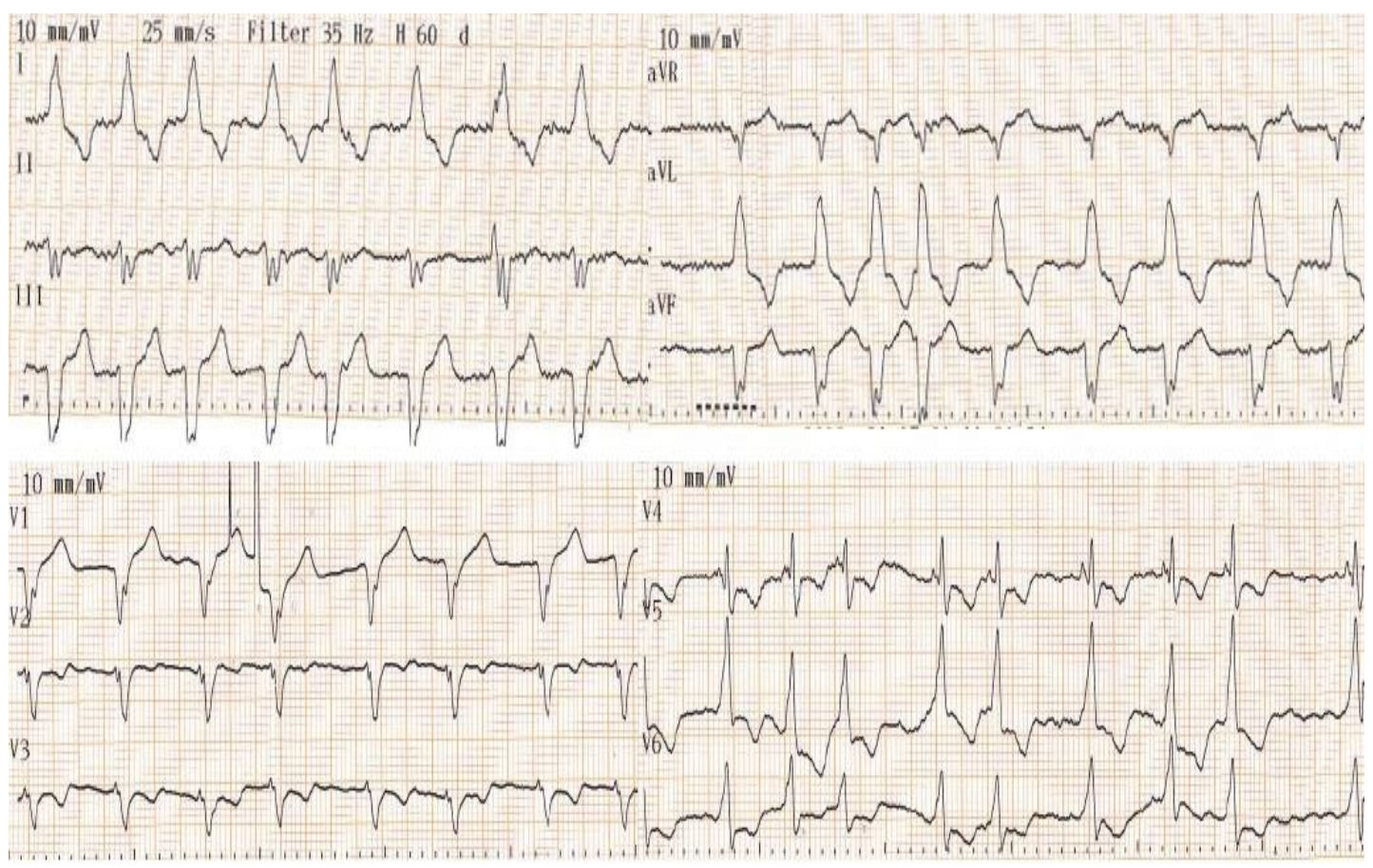

Figura 1. Electrocardiograma. Ritmo de fibrilación auricular, bloqueo de rama izquierda del haz de His.

Los hallazgos relevantes en el ecocardiograma fueron: ventrículo izquierdo dilatado $\left(36 \mathrm{~mm} / \mathrm{m}^{2}\right)$ con hipertrofia excéntrica moderada, acinesia de los segmentos apicales, acinesia media y apical del séptum anterior, hipocinesia severa del séptum inferior y demás paredes hipocinéticas; adelgazamiento del séptum interventricular (en relación con intervención quirúrgica previa); prótesis mecánica mitral normo funcionante; dilatación severa de las cavidades derechas e hipertrofia de ventrículo derecho; dilatación severa de la aurícula izquierda. La función sistólica biventricular se encontró severamente comprometida, fracción de eyección del ventrículo izquierdo (FEVI) de $30 \%$ y la función longitudinal del ventrículo derecho -estimada por el desplazamiento sistólico del anillo tricúspideo (TAPSE)- de 9,0 mm. En resumen, los hallazgos ecocardiográficos descartaron prótesis disfuncional y se evidenció falla biventricular severa, con dilatación de todas las cavidades e hipertrofia biventricular.

Se inició ventilación mecánica no invasiva, terapia diurética (furosemida), antiarrítmica (amiodarona intravenosa y en infusión), control de frecuencia cardiaca (digoxina intravenosa). La resonancia magnética cardiaca reportó gran hipertrofia biventricular con dilatación de todas las cavidades cardiacas y se descartaron otras etiologías como embolia pulmonar, cardiopatías infiltrativas o enfermedad isquémica cardiaca (figura 2). 
Esta evolución de cardiomiopatía hipertrófica a fase dilatada-hipocinética se configuró como etapa "burnout" y denota un punto de quiebre en el pronóstico, generalmente progresando a insuficiencia refractaria, en donde la estrategia terapéutica definitiva es el trasplante.

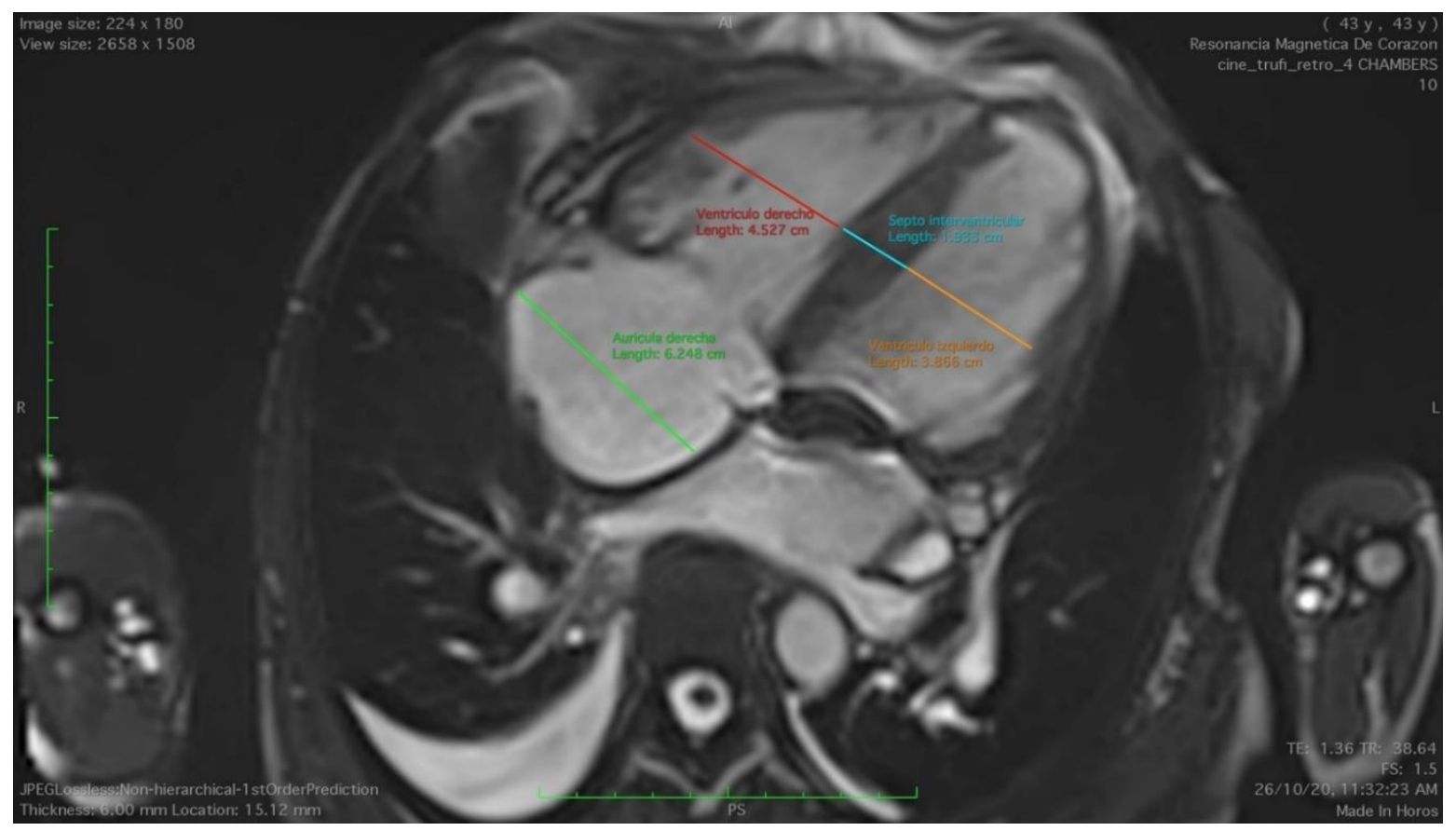

Figura 2. Resonancia magnética cardiaca. Dilatación de cavidades cardiacas asociado a hipertrofia biventricular.

Durante la hospitalización se realizó compensación de la falla cardiaca (con espironolactona, metoprolol tartrato, furosemida, hierro parenteral), de las comorbilidades y control de frecuencia cardiaca para la fibrilación auricular (metoprolol tartrato y digoxina). Se realizó junta médica con el servicio de Cardiología-Electrofisiología y se determinó realizar cardioversión eléctrica seguida de tratamiento oral con amiodarona, el cual fue exitoso. Por lo anterior, se dio egreso de la institución para continuar tratamiento ambulatorio de falla cardiaca con inhibidor de la enzima convertidora de angiotensina, betabloqueador, espironolactona y diurético. Se dejó warfarina para el manejo de su fibrilación auricular asociada a prótesis mecánica mitral con valor del índice internacional normalizado (INR, por sus siglas en inglés) entre 2,5 a 3,5. 
Fue valorada nuevamente luego de un mes del alta con nuevos estudios: ecocardiografía transesofágica y Holter, con resultados que fueron alentadores puesto que se mantuvo en ritmo sinusal y la fracción de eyección del ventrículo izquierdo mejoró a 40 \%, permaneciendo la dilatación de cavidades y ahora con clase funcional I de la (NYHA). La paciente manifestó adecuada adherencia farmacológica que se correlacionaba con su mejoría clínica. Finalmente, en decisión conjunta se da orden para realizar aislamiento de venas pulmonares y adición de un inhibidor del cotransportador sodio-glucosa tipo 2 (SGLT2i, por sus siglas en inglés). A la fecha, se encuentra en seguimiento multidisciplinario para definir si es candidata a un trasplante o dispositivo cardíaco.

\section{Discusión}

La cardiomiopatía hipertrófica se define como una hipertrofia del ventrículo izquierdo $>1,5 \mathrm{~cm}$ en ausencia de enfermedades cardiovasculares que expliquen los cambios como hipertensión arterial, valvulopatías, enfermedad isquémica, entre otros ${ }^{(1,2)}$. Los pacientes suelen estar asintomáticos; sin embargo, el espectro de presentación es amplio y puede encontrarse obstrucción del tracto de salida del ventrículo izquierdo, disfunción sistólica y síntomas de falla cardiaca, arritmias como la fibrilación auricular, muerte súbita y la etapa final o burnout. Esta diferencia en su presentación se ha relacionado con la existencia de otros factores de riesgo que precipitan la aparición de síntomas y evolución de la enfermedad ${ }^{(2,3)}$.

La prevalencia de la cardiomiopatía hipertrófica en Colombia es del 2,3 \% y se relaciona principalmente con dolor torácico e insuficiencia mitral como hallazgos al momento del diagnóstico. No se cuenta con datos sobre la prevalencia de fenotipo de burnout en el país, y se estima que a nivel mundial podría encontrarse entre $5 \%$ a $10 \%$ de los pacientes con

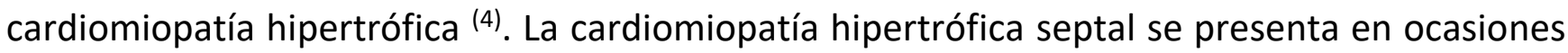
con obstrucción del tracto de salida del ventrículo izquierdo, asociándose con la severidad de los síntomas y el desarrollo de alteraciones estructurales secundarias, como valvulopatías y $\operatorname{arritmias}^{(1,4,5)}$. La evaluación morfológica inicial es realizada por medio de ecocardiograma transtorácico, aunque la resonancia magnética cardiaca es el estándar de oro, además de aportar valor pronóstico ${ }^{(1,6,7)}$.

El fenotipo de "burnout" se presenta hasta en el $10 \%$ de los pacientes con cardiomiopatía hipertrófica. Es característico el realce tardío de gadolinio, además de dilatación de cavidades cardiacas e hipertrofia septal, apical o difusa ${ }^{(1,5-7)}$. A pesar de no cursar con obstrucción del tracto de salida del ventrículo izquierdo, los pacientes tienen un peor pronóstico debido a que 
sus cavidades se encuentran dilatadas, por lo que se incrementa el riesgo de arritmias. El pronóstico adverso también puede estar relacionado con disfunción sistólica e hipertensión pulmonar secundaria ${ }^{(3)}$.

El tratamiento depende de la presentación de la enfermedad y está encaminado a reducir los síntomas y mejorar la calidad de vida. El manejo médico para la falla cardiaca y el control de arritmias pueden mejorar la clase funcional y reducir el riesgo de eventos embólicos, respectivamente. El uso de bloqueadores de canales de calcio debe evitarse en pacientes con fracción de eyección del ventrículo izquierdo menor de 40 \% o con obstrucción del tracto de salida del ventrículo izquierdo, debido a que el efecto vasodilatador puede empeorar la obstrucción ${ }^{(1,4,5)}$.

Se encuentran en curso nuevas terapias que buscan mejorar la sintomatología y supervivencia, entre ellos el Mavacamten, cuya función es inhibir la miosina cardíaca (10-12). También la Perhexilina, que induce cambios en el metabolismo celular, reduciendo el consumo de ácidos grasos y favoreciendo el uso de carbohidratos como sustrato metabólico ${ }^{(1,13)}$.

El manejo invasivo está indicado en pacientes sintomáticos (NYHA III/IV) a pesar de la terapia médica óptima con un gradiente del tracto de salida del ventrículo izquierdo de $50 \mathrm{~mm} / \mathrm{Hg}$ (en reposo o provocado). Las opciones terapéuticas son ablación septal con alcohol o miectomía septal ${ }^{(3,5,14,15)}$. En lo que concierne a la fibrilación auricular asociada, se encuentra hasta en el $32 \%$ de los casos, siendo considerado un factor de riesgo independiente para mortalidad. Se ha descrito recientemente la utilidad de realizar ablación de los focos de la arritmia en el momento de realizar la miectomía como estrategia para mantener el ritmo a largo plazo y disminuir la mortalidad asociada ${ }^{(16-20)}$.

Es llamativo del caso que, a pesar de haber realizado un manejo médico y quirúrgico oportuno, la paciente presentó una progresión de la enfermedad hasta desarrollar el fenotipo de burnout. Lo anterior, sugiere que existen mecanismos que podrían favorecer el desarrollo de dilatación de cavidades cardiacas a pesar de la intervención de la enfermedad. Se ha demostrado la estrecha relación de genes implicados en el desarrollo de la cardiomiopatía hipertrófica y la cardiomiopatía dilatada, entre ellos MYBPC3, MYH6, MYH7 y ACTN2 ${ }^{2}$.

Hay pocos casos reportados de cardiomiopatía hipertrófica en fase de burnout lo que podría estar en relación a un subregistro. Recientemente Caldeira et al. reportan una paciente con cardiomiopatía hipertrófica con fenómeno de burnout y por medio de pruebas genéticas 
determinan la presencia de una doble mutación de la proteína $C$ que se une a la miosina truncada (MYBPC3; mutación c.2827C>T en el exón 27; p. Arg943X), previamente descrita y asociada con miocardiopatía hipertrófica, y una nueva mutación de la cadena pesada de la beta miosina (MYH7; mutación c.2821C>T en el exón 23; p.Arg941Cys). La paciente recibió manejo para falla cardiaca, tal como ocurrió en la paciente descrita, e implante de desfibrilador ${ }^{(21)}$.

Las complicaciones tardías de la cardiomiopatía hipertrófica incluyen este fenómeno de agotamiento o burnout y arritmias como la fibrilación auricular, por lo que algunos tratamientos a considerar serían la terapia de resincronización cardiaca, el desfibrilador implantable o el aislamiento de venas pulmonares, este último ofrecido a la paciente ${ }^{(5)}$. Esto como puente al trasplante cardiaco, el cual presenta una sobrevida del $92 \%$ a cinco años $(2,3,5,22)$.

\section{Conclusión}

La cardiomiopatía hipertrófica es una entidad frecuente, pero subdiagnosticada. Si bien es una entidad ya conocida, sus complicaciones tardías son poco descritas. Esto dispone implicaciones cruciales desde el punto de vista terapéutico y pronóstico, por lo que conocerlas lleva a un diagnóstico y tratamiento oportuno que impacta finalmente en la calidad de vida y mortalidad. El trasplante cardiaco representa una opción terapéutica muy importante en aquellos pacientes que han progresado al burnout, siendo actualmente la única alternativa de manejo eficaz. Finalmente, este caso aporta diferentes estrategias para implementar a lo largo del curso de la enfermedad.

\section{Conflictos de interés y financiación}

Ninguno de los autores tiene conflictos de interés o recibió remuneración alguna durante la escritura, edición o publicación de este artículo.

\section{Consentimiento informado}

Se obtuvo consentimiento informado de la paciente.

\section{Bibliografía}

1. Antunes M de O, Scudeler TL. Hypertrophic cardiomyopathy. IJC Hear Vasc. 2020;27:100503.

2. Teekakirikul P, Zhu W, Huang HC, Fung E. Hypertrophic cardiomyopathy: An overview of genetics and management. Biomolecules. 2019;9(12):1-11. 
3. Maron BJ. Clinical Course and Management of Hypertrophic Cardiomyopathy. N Engl J Med. 2018;379(7):655-68.

4. Brinkley DM, Wells QS, Stevenson LW. Avoiding Burnout from hypertrophic cardiomyopathy. J Am Coll Cardiol. 2020;75(24):3044-3047. doi: 10.1016/j.jacc.2020.05.009. PMID: 32553257.

5. Ommen SR, Mital S, Burke MA, Day SM, Deswal A, Elliott P, et al. 2020 AHA/ACC Guideline for the diagnosis and treatment of patients with hypertrophic cardiomyopathy: executive summary: A report of the American College of Cardiology/American Heart Association Joint Committee on Clinical Practice Guidelines. Circulation. 2020;533-57.

6. Maron MS, Rowin EJ, Maron BJ. How to image hypertrophic cardiomyopathy. Circ Cardiovasc Imaging. 2017;10(7):1-15.

7. Abad P, Asociacion Colombiana de Radiologia. Miocardiopatías. Dr. Pedro Abad Díaz Conferencia Virtual [Internet]. Colombia: Asociacion Colombiana de Radiologia; 2018. Available from: http://www.acronline.org/ACR-Admin/NOTICCR/ArtMID/1061/ArticleID/5966/Miocardiopat237as-Dr-Pedro-Abad-D237azConferencia-Virtual

8. Heitner SB, Jacoby D, Lester SJ, Owens A, Wang A, Zhang D, et al. Mavacamten treatment for obstructive hypertrophic cardiomyopathy a clinical trial. Ann Intern Med. 2019;170(11):741-8.

9. Zhang YP, He B. Mavacamten: a promising new target drug for the treatment of hypertrophic cardiomyopathy. Zhonghua Xin Xue Guan Bing Za Zhi [Internet]. 2021 Apr 24;49(4):310-3. Available from: http://www.ncbi.nlm.nih.gov/pubmed/33874678

10. Olivotto I, Oreziak A, Barriales-Villa R, Abraham TP, Masri A, Garcia-Pavia P, et al. Mavacamten for treatment of symptomatic obstructive hypertrophic cardiomyopathy (EXPLORER-HCM): a randomised, double-blind, placebo-controlled, phase 3 trial. Lancet [Internet]. 2020;396(10253):759-69. Available from: https://linkinghub.elsevier.com/retrieve/pii/S014067362031792X

11. Tower-Rader A, Ramchand J, Nissen SE, Desai MY. Mavacamten: a novel small molecule modulator of $\beta$-cardiac myosin for treatment of hypertrophic cardiomyopathy. Expert Opin Investig Drugs [Internet]. 2020;29(11):1171-8. Available from: https://www.tandfonline.com/doi/full/10.1080/13543784.2020.1821361 
12. Ho CY, Mealiffe ME, Bach RG, Bhattacharya M, Choudhury L, Edelberg JM, et al. Evaluation of Mavacamten in symptomatic patients with nonobstructive hypertrophic cardiomyopathy. J Am Coll Cardiol [Internet]. 2020;75(21):2649-60. Available from: https://linkinghub.elsevier.com/retrieve/pii/S0735109720348567

13. Cooper RM, Raphael CE, Liebregts M, Anavekar NS, Veselka J. New developments in hypertrophic cardiomyopathy. Can J Cardiol [Internet]. 2017;33(10):1254-65. Available from: https://linkinghub.elsevier.com/retrieve/pii/S0828282X17303719

14. Nishimura RA, Seggewiss H, Schaff H V. Hypertrophic obstructive cardiomyopathy: Surgical myectomy and septal ablation. Circ Res. 2017;121(7):771-83.

15. Solomon Z, Breton C, Rowin EJ, Maron BJ, Maron MS, Chen FY, et al. Surgical Approaches to hypertrophic obstructive cardiomyopathy. Semin Thorac Cardiovasc Surg. 2018;30(2):125-8.

16. O'Neill L, Duytschaever M, Knecht S. Atrial fibrillation ablation in patients with hypertrophic cardiomyopathy: do not throw in the towel too fast! Europace [Internet]. 2021; Available from: http://www.ncbi.nlm.nih.gov/pubmed/33930128

17. Ali A. Atrial Fibrillation in Hypertrophic Cardiomyopathy: Diagnosis and Considerations for Management. J Atr Fibrillation [Internet]. 2018;10(5). Available from: http://www.jafib.com/published.php?type=full\&id=1556

18. Santangeli P, Di Biase L, Themistoclakis S, Raviele A, Schweikert RA, Lakkireddy D, et al. Catheter Ablation of atrial fibrillation in hypertrophic cardiomyopathy. circ arrhythmia electrophysiol [Internet]. 2013;6(6):1089-94. Available from: https://www.ahajournals.org/doi/10.1161/CIRCEP.113.000339

19. Cui H, Schaff H V., Dearani JA, Lahr BD, Viehman JK, Geske JB, et al. Does ablation of atrial fibrillation at the time of septal myectomy improve survival of patients with obstructive hypertrophic cardiomyopathy? J Thorac Cardiovasc Surg [Internet]. 2021;161(3):997-1006.e3. Available from: https://linkinghub.elsevier.com/retrieve/pii/S0022522320324545

20. Bogachev-Prokophiev A V., Afanasyev A V., Zheleznev SI, Pivkin AN, Fomenko MS, Sharifulin RM, et al. Concomitant ablation for atrial fibrillation during septal myectomy in patients with hypertrophic obstructive cardiomyopathy. J Thorac Cardiovasc Surg [Internet]. 2018;155(4):1536-1542.e2. Available from: https://linkinghub.elsevier.com/retrieve/pii/S0022522317318251 
21. Caldeira D, Lopes LR, Cruz I, Almeida AR, Morgado G, Gomes C, Stuart B, Almeida S, Brandão L, Pereira $\mathrm{H}$. The burnout stage of an apical hypertrophic cardiomyopathy. Int J Cardiol. 2014;177(3):e179-80. doi: 10.1016/j.ijcard.2014.08.092. Epub 2014 Aug 23. PMID: 25179554.

22. Rowin EJ, Maron BJ, Abt P, Kiernan MS, Vest A, Costantino F, et al. Impact of Advanced therapies for improving survival to heart transplant in patients with hypertrophic cardiomyopathy. Am J Cardiol. 2018;121(8):986-96. 